\title{
Ein neuer Name, ein neues Profil - Infusion Therapy and Transfusion Medicine wird zu Transfusion Medicine and Hemotherapy
}

Im Jahre 1973 wurde die Zeitschrift INFUSIONSTHERAPIE von namhaften Klinikern als deutschsprachige Fachzeitschrift gegründet. Die Zeitschrift behandelte überwiegend klinische und klinisch-theoretische Themen zur Infusionstherapie sowie spezielle Fragen der klinischen Ernährung. Die wissenschaftlichen und klinischen Fragestellungen aus der Transfusionsmedizin waren anfangs nur selten Thema in der InFusionsTHERAPIE. Erst Anfang der 1980er Jahre wurde die Bedeutung neuer Risiken durch Blutprodukte und deren verheerende Auswirkungen auf die Sicherheit der Blutversorgung immer deutlicher. Parallel hierzu wuchs auch der Anteil spezieller transfusionsmedizinischer Beiträge, wobei insbesondere aktuelle Fragen zu Risiken und Sicherheit der Blutversorgung im Vordergrund standen. Ende der 1980er Jahre wurde INFUSIONSTHERAPIE unter dem Namen INFUSIONSTHERAPIE UND Transfusionsmedizin auch das wichtigste offizielle Publikationsorgan der Deutschen Gesellschaft für Transfusionsmedizin und Immunhämatologie (DGTI).

Die wissenschaftliche Entwicklung in der Transfusionsmedizin und die Folgen der europäischen Vereinigung hatten Einfluss auch auf die nationale Blutversorgung. Deshalb war die Forderung nach einer internationalen Ausrichtung der Zeitschrift richtig und notwendig, um auch im europäischen Sprachraum breit vertreten zu sein. Die Namensänderung in INFUSION THERAPY AND TRANSFUSION MEDICINE im Jahr 2000 und die Veröffentlichung von Originalartikeln in englischer Sprache nach unabhängiger Fachbegutachtung unterstützten diese Neuorientierung. Ein besonderes Anliegen der Zeitschrift war es dabei immer, ihren Lesern sowohl fachmedizinische als auch wissenschaftliche, medikolegale und ökonomische Themen von der Blutspende bis zur komplexen Hämotherapie klar und verständlich zu präsentieren. Um diesem Anspruch auf hohem Niveau auch unter zukünftig noch schwierigeren Rahmenbedingungen gerecht werden zu können, haben Herausgeber, Schriftleitung und Verlag einvernehmlich mit dem Vorstand der DGTI beschlossen, das fachmedizinisch-wissenschaftliche Profil der Zeitschrift den aktuellen Bedürfnissen und Erfordernissen noch deutlicher anzupassen. Diese stärkere klinische Ausrichtung der Zeitschrift wird auch in ihrem neuen Namen ausgedrückt: TRANSFUSION MEDICINE AND HEMOTHERAPY.

Zukünftig wird Transfusion Medicine And HeMOTHERAPY klinisch orientierte Themen und aktuelle wissenschaftliche Entwicklungen auf dem Gebiet der klinischen Transfusionsmedizin und Hämotherapie noch stärker berücksichtigen. Die Herausgeber sehen in ihrer Entscheidung keinen Bruch mit der klinischen und klinisch-theoretischen Tradition der INFUSIONSTHERAPIE bZW. INFUSIONSTHERAPIE UND TRANSFUSIONSMEDIZIN, die in diesem Jahr ihren 30. Geburtstag feiern darf. Im Gegenteil, die Richtungsentscheidung, klinische Aspekte der Transfusionsmedizin und Hämotherapie wieder stärker zu berücksichtigen, ist die Konsequenz einer erfolgreichen Arbeit der Transfusionsmedizin in den vergangenen 30 Jahren. Die wissenschaftlichen Erfolge auf dem Gebiet Transfusionsmedizin haben heute wesentlichen Anteil am hohen Sicherheitsstandard, der bei der Herstellung und Anwendung von Blutprodukten erreicht wird.

Die Verantwortlichen hoffen, dass die neue Konzeption von Transfusion Medicine and Hemotherapy sowohl von den Lesern als auch von Autoren mit großem Interesse und $\mathrm{Zu}$ stimmung aufgenommen wird. Sie wünschen sich eine aktive Beteiligung der Ärzte und Wissenschaftler, die auf dem interdisziplinären Gebiet der Transfusionsmedizin und Hämotherapie tätig sind.

Abschließend möchten die Verantwortlichen all denen danken, die mit ihrer Arbeit ganz wesentlich dazu beigetragen haben, dass die erfolgreiche fachmedizinische und wissenschaftliche Entwicklung der Transfusionsmedizin in den letzten 30 Jahren auch durch eine kompetente Fachzeitschrift begleitet und dokumentiert werden konnte. Ohne diese grundlegenden Vorarbeiten der früheren Herausgeber und Schriftleiter wäre eine Fortsetzung der erfolgreichen Arbeit mit neuen Inhalten und Schwerpunkten unter dem neuen Namen Transfusion Medicine AND Hemotherapy kaum zu bewältigen gewesen.

Für die Herausgeber W. Sibrowski, Münster 